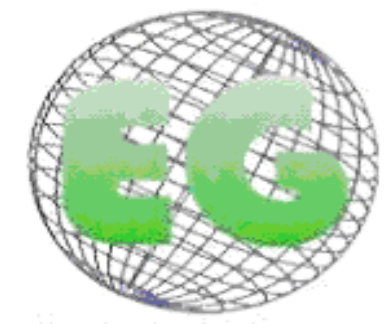

ISSN 1695-6141 N 27

\title{
Entrevista con gestores como método pedagógico para la gerencia en enfermeria: conocer para ser
}

Entrevista com gestores como método pedagógico para o gerenciamento na enfermagem: conhecer para ser

\section{${ }^{*}$ Ferreira Júnior, AR., ${ }^{* *}$ de Souza Vieira, LJE., ${ }^{* * *}$ de Barros, NF.}

\author{
*Universidade Estadual de Campinas - UNICAMP. E-mail: junioruruoca@hotmail.com **Universidade de \\ Fortaleza - UNIFOR ****Universidade Estadual de Campinas. Brasil.
}

Palabras clave: gestión en salud; educación superior; educación en enfermería.

Palavras-chave: gestão em saúde; ensino superior; educação em enfermagem.

Keywords: Health Management; Higher Education; Nursing Education.

\section{RESUMEN}

El articulo discute metodologías activas de enseñanza-aprendizaje sobre la gerencia en enfermería, por medio de un relato de experiencia que sucedió en la Institución de Educación Superior, en Sobral - Ceará - Brasil, en 2010. Entrevistas a enfermeros-gestores de diversas áreas son presentadas como herramienta pedagógica propiciadoras de discusiones acerca de asuntos transversales al tema de gestión en salud. Hubo experimentación de la construcción y se implementó la entrevista como técnica de colecta de datos por los alumnos y las temáticas discutidas en la sala de aula enfatizaron las políticas nacionales de salud pública, así como las potencialidades del enfermero como gestor en los servicios de salud. Se observó el beneficio de la integración de conocimientos entre la teoría y la práctica, contribuyendo al reconocimiento de las dificultades y desafíos a ser enfrentados por los futuros profesionales.

\section{RESUMO}

$\mathrm{O}$ artigo discute metodologias ativas de ensino-aprendizagem do gerenciamento em Enfermagem, por meio de relato de experiência ocorrida em Instituição de Educação Superior, em Sobral - Ceará - Brasil, em 2010. Entrevistas a enfermeiros gestores de áreas diversas são apresentadas como ferramenta pedagógica propiciadora de discussões acerca de assuntos transversais ao tema da gestão em saúde. Ocorreu experimentação da construção e implementação da entrevista como técnica de coleta de dados pelos discentes e as temáticas discutidas na sala de aula enfatizaram as políticas nacionais de saúde pública, bem como as potencialidades do enfermeiro como gestor nos serviços de saúde. Observou-se o favorecimento da integração de conhecimentos entre a teoria e a prática, contribuindo com o reconhecimento das dificuldades e desafios a ser enfrentados pelos futuros profissionais. 


\section{ABSTRACT}

This article discusses the active teaching-learning methodologies of management in nursing, through reports of an experiment performed in a Higher Education Institution, in Sobral - Ceará - Brazil, in 2010. Interviews with nurses from different fields are presented as a pedagogical tool that enables discussions about transverse topics to Health management. It was done an experiment on the construction and application of interviewing by the students as a data collecting technique; furthermore, the topics discussed in class emphasized the national policies on Public Health, as well as the potentialities of the nurse as a manager for Health services. It could be observed a favouring of knowledge integration between theory and practice, which contributes for the recognizement of troubles and challenges that are to be faced by the future professionals.

\section{INTRODUCCIÓN}

La Gerencia en Enfermería está aumentando, de manera notable, como área dentro de la profesión que agrega profesionales pro-activos, exigiendo de la academia transformaciones que integren competencias, habilidades y valores, al alcance de una praxis eficaz. Fomentar la intersección de la teoría ${ }^{1}$ y de la práctica durante la formación académica es una posibilidad de devolver a la sociedad profesionales con mayor éxito para enfrentar los desafíos del mercado de trabajo.

De esta manera, el cambio de actitud de los educadores beneficia a todos, ya que trae innovaciones al proceso de enseñanza y, especialmente, hace que este se torne placentero, singular y repleto de significaciones positivas para las personas involucradas. Existe la defensa de un modelo de enseñanza en el cual el discente intercambia experiencias de forma continua con el docente, adquiriendo éste el papel de facilitador y propiciador de ambientes favorables para que sobrevengan discusiones y diálogos ${ }^{2}$.

Entendemos que el monólogo y la unilateralidad en la formación académica no favorecen la mediación de conflictos y tampoco la toma de decisiones, herramientas necesarias para la gestión, ante la turbulencia de la post-modernidad, entendida como la forma de modernidad que trasciende a la propia modernidad, denotando que los conocimientos anteriores pueden siempre generar otros ${ }^{3}$.

La sociedad ambiciona transformaciones en las formas de enseñar salud en Brasil, pues en la mayoría de las instituciones los gestores de educación están distantes de los gestores de la salud, dificultando la comunicación. El aprendizaje está centrado en el profesor, en los libros y en los textos, cuando debería centrarse en el intercambio de experiencias de uno con los que ya actúan en el área ${ }^{4}$.

La metodología activa debe estar en el centro de las nuevas construcciones para la formación de buenos profesionales, considerando que conocer es transformar y esto solo se vuelve provechoso a partir del momento en que hay respeto a la autonomía por parte del binomio docente/discente, en relación al proceso de producción y adquisición de ese conocimiento ${ }^{5}$.

Distintas competencias deben ser trabajadas en la formación del enfermero, siendo estas: atención a la salud, toma de decisiones, comunicación, liderazgo, administración y gerencia y educación permanente ${ }^{6}$. El concepto de competencias es delimitado por diferentes dimensiones, siendo las habilidades, los conocimientos y las prácticas, las más importantes ${ }^{7}$. Esto debe posibilitar vivencias del futuro enfermero en su campo de actuación, no solo en lo que se refiere a la aproximación con la teoría, sino también agregando a eso, la experiencia práctica, porque aún no existe un consenso entre las competencias gerenciales específicas 
de un enfermero, aún así debe haber un estímulo para proponer debates sobre esta construcción, basándose en la práctica de los profesionales para, además de definirlas, desarrollarlas en los discentes ${ }^{8}$.

Existen críticas constantes dirigidas al modelo funcional de enseñanza ${ }^{2}$, priorizando un modelo dirigido direccionado al intercambio de experiencias entre la academia y los servicios de salud, facilitando la construcción individual y colectiva del conocimiento. El proceso de enseñar debe propiciar una discusión provechosa sobre las dificultades intrínsecas que envuelven el tema, afectando a la importancia de la convergencia de conceptos, dificultades y desafíos en la interface entre la Enfermería y la gestión, demostrando los diversos aspectos y la complejidad de estos.

Con miras a respetar lo que dicta la Ley de Directrices y Bases de la Educación Nacional (LDB) - Ley no 9,394 de 20 de diciembre de 1996, que adoptó las directrices curriculares específicas para cada curso, la Resolución del Consejo Nacional de Educación nº 03/2001 tratando de las directrices curriculares para la graduación en Enfermería de Brasil, el Programa Nacional de Reorientación de la Formación Profesional en Salud (Pro-Salud) y programas relacionados que reorientan la formación del profesional de la salud, la Enfermería avanza con nuevas propuestas curriculares. La reformulación de estas propuestas integra contenidos y facilita la comprensión del "todo"; se configura como estrategia para romper con el modelo dicotómico y fragmentado seguido por muchas Instituciones de Enseñanza Superior ${ }^{9-13}$.

Y nada más pertinente que conducir al alumno para ser sujeto de su aprendizaje en la adquisición de competencias que trascienden a libros y textos, siendo fructífero el intercambio de experiencias y la aproximación con la práctica. En este sentido, este trabajo relata el uso de una tecnología pedagógica que coloca a los discentes en contra de construcciones realizadas en la academia con la práctica de profesionales reconocidos en el área de gestión en Enfermería.

Este asume como tecnología pedagógica las nuevas maneras de enseñar (¿y por qué no, de cuidar?) ${ }^{14}$ que se dirigen al encuentro de una gestión competente, de calidad, en la que la objetividad asume la importancia de las subjetividades para el éxito de la gerencia del cuidado.

\section{MÉTODO}

En este contexto, en el que creemos en el potencial de los individuos, relatamos una experiencia con el empleo de metodologías activas, llevada a cabo en el municipio de Sobral - Ceará - Brasil, en el año 2010, construida en la disciplina de Organización de los Servicios de Salud, que integra la propuesta curricular del Curso de Graduación en Enfermería de una Institución de Enseñanza Superior - IES, de ámbito privado.

Esta metodología procuró favorecer a los discentes en la confrontación de las construcciones realizadas en el campo teórico con la práctica experimentada por profesionales reconocidos como gestores en Enfermería, en la región Norte de Ceará.

Múltiples discusiones en el aula fomentaron la construcción y comprensión de los conceptos referentes a la gestión en salud, con explicaciones teóricas contextualizadas ${ }^{15-17}$, sin perder de vista las especificidades que circunscriben a la gestión en el campo de la Enfermería. 
Se hacen necesarias construcciones en el aula que discutan el proceso de trabajo y la forma de gerenciamiento en Enfermería, buscando la utilización de nuevas herramientas y tecnologías con la intención de innovar y modificar la profesión ${ }^{18}$.

La enseñanza para enfermeros, necesita contar con estrategias pedagógicas innovadoras, especialmente en el área de administración en salud que requiere competencias específicas para la formación de buenos profesionales y ciudadanos ${ }^{19}$.

Siguiendo esta propuesta, se realizó la división de los estudiantes en seis grupos de ocho personas que se responsabilizaron de contratar enfermeros de reconocida experiencia en el área de gerencia en la región Norte del Estado de Ceará.

En grupo, se definió que la estrategia para facilitar las discusiones sería la utilización de entrevistas a los gestores. Esto hizo que fuese conocida la realidad vivida por ellos de una manera menos formal, como también favoreció un dialogo entre los enfermeros de éxito profesional reconocido y los alumnos en formación.

La literatura certifica que la entrevista es una técnica privilegiada de comunicación, pues se trata de un diálogo destinado a construir informaciones que sean relevantes para los interlocutores. Puede ser utilizada como sondeo inicial para el conocimiento de algún asunto, pero también como técnica importante de investigación ${ }^{20-21}$. Las entrevistas son bastante utilizadas en al área de la salud como herramienta flexible y poderosa para discutir sobre los comportamientos y prácticas ${ }^{22}$.

Así, los grupos elaboran guiones para las entrevistas semi-estructuradas, con preguntas acerca de los desafíos, dificultades y facilidades encontradas por los profesionales en su práctica diaria.

Cada equipo se hizo responsable de un área de actuación diferente de la gerencia en Enfermería, orientando y conociendo los distintos cargos, tal como: Director de hospital, Secretario municipal de salud, Gerente de la Unidad Básica de Salud, Coordinador de curso de graduación, Gerente del centro quirúrgico y Gerente del cuerpo de Enfermería de un hospital. El profesional era invitado a participar en una clase en la institución de enseñanza, donde serían realizadas las preguntas formuladas por el equipo responsable, conforme el cronograma establecido.

Estos eran momentos ricos en intercambio de experiencias, cuando el entrevistado conversaba sobre su praxis, informando de las características profesionales que él creía que eran indispensables para su ideal de trabajo.

También se trataban otros aspectos sobre la apreciación de la Enfermería en la sociedad debido al ascenso de los profesionales, especialmente en el área de gestión de los servicios de salud. El marketing personal del enfermero aparecía como tema transversal en las conversaciones, siendo la herramienta más eficiente en el ambiente profesional para posibilitar que sus pensamientos, actitudes, presentación y comunicación trabajen a su favor $^{23}$. 


\section{RESULTADOS}

\section{Conociendo la gestión por medio de la experiencia de gestores}

Las distintas actuaciones profesionales encontradas hicieron posible reducir las dudas de los alumnos sobre la práctica de estos enfermeros, generando discusiones sobre las políticas nacionales, la legislación vigente en el futuro de las acciones que incluían esas áreas de gestión.

El secretario municipal de salud, trajo importantes asuntos para la discusión, enfocando su papel como gestor del Fondo Municipal de Salud y del Sistema Único de Salud - SUS en el ámbito de su municipio de actuación.

El enfermero es una de las categorías profesionales que ocupa funciones importantes para la implementación del SUS. Y un diferencial es que a pesar de encontrarse ante situaciones complejas, las actuaciones técnicas sobresalgan en detrimento de las políticas ${ }^{24}$.

El proceso de gestión en salud, está en continuo cambio, ya que las políticas son construidas y perfeccionadas constantemente y esto puede ser experimentado con la implantación del Pacto por la Salud, con las dimensiones del Pacto por la vida, de Gestión y en Defensa del SUS, que modificó notablemente los mecanismos para la implementación de acciones y evaluación de los resultados ${ }^{25}$.

Varias herramientas de Enfermeria ${ }^{26}$ fueron colocadas como utilizadas en el proceso de gerenciamiento del SUS en el ámbito municipal, tales como: observación, planificación, creatividad, conocimiento, organización y habilidad en la articulación.

Esto hace posible la toma de decisiones mejor construidas, generando impactos positivos, considerando las diversas responsabilidades del gestor municipal, tales como: ordenación y aplicación de los recursos financieros del Fondo Municipal de Salud, gerencia de los recursos humanos, participación en el proceso de licitaciones y compras, planificación estratégica en el área de salud, así como fortalecimiento de los equipos para el funcionamiento continuo de los servicios ${ }^{27}$.

Vale la pena destacar que el enfermero, en su formación discute y construye conceptos que influirán su praxis, como el de trabajo en equipo, liderazgo y compromiso en defensa del SUS.

Los mayores desafíos expuestos por el secretario de salud fueron las acciones que exigían conceptos de las áreas de Derecho y Contabilidad, visto que hay necesidades del gestor de trabajar constantemente utilizando estas herramientas con la intención de ejecutar las acciones y responder de aquellas que fueron realizadas, siempre siguiendo las leyes y normativas que rigen la administración pública, editadas por el Ministerio de Salud y el Tribunal de Cuentas de los Municipios, por ejemplo.

El gerente de la Unidad Básica de Salud, integrante de la Estrategia Salud de la Familia, procuró destacar la importancia de la integración entre servicio y comunidad, siendo ese uno de los principios defendidos en el SUS.

Fue también fuertemente discutida la Política Nacional de la Atención Básica y la Política Nacional de Promoción de Salud, dado que son orientadoras de las acciones desarrolladas por los enfermeros de todo el país. Muy importantes para el entendimiento de la situación 
actual del área de salud en Brasil, puesto que la atención primaria, con la búsqueda de la optimización de la promoción de salud individual y colectiva está siendo colocada como prioritaria $^{28-29}$.

Son variadas las contribuciones de los enfermeros gerentes en la atención primaria, ya que son capaces de potenciar los cuidados prestados de forma colectiva a las familias que generalmente están adscritas a las unidades de salud. Las principales dificultades enunciadas son referentes a los parcos recursos financieros y humanos, así como la necesidad constante de actualización profesional ${ }^{30-32}$.

Los enfermeros del área hospitalaria trajeron debates pertinentes a la práctica específica en este ambiente de cuidado. El Director del hospital, el Gerente del centro quirúrgico y el Gerente del cuerpo de Enfermería eran de instituciones diferentes, de ámbito público, privado y filantrópico, y pudieron dar a todos una visión más específica sobre el trabajo en estos centros.

El enfermero debe estar preparado para afrontar las nuevas demandas y esto queda explícito en los cuidados médicos hospitalarios, dado que son generalmente realizados en una situación de mayor fragilidad para el usuario. La preparación profesional es importante, pues se tiene la necesidad de actuación ética, mirando por la calidad de los servicios prestados y utilizando varias herramientas de gestión, tales como: innovación, creatividad y liderazgo en el gerenciamiento de los equipos para que todo ocurra de la mejor forma posible ${ }^{33-36}$.

Ya la Coordinadora del Curso de Graduación hizo posible la construcción de reflexiones bien diferenciadas acerca del ámbito de la gestión en Enfermería, problematizando junto a los discentes, diferentes cuestiones pedagógicas, de la relación docente e institucional, así como de cualquiera de los asuntos referentes a la práctica de la enseñanza y de la formación de los futuros enfermeros.

Los gestores de los cursos de graduación en el área de salud tienen también que estar atentos a las nuevas formas de enseñanza articulada con las modificaciones oriundas del Sistema Único de Salud, dado que es necesaria una formación menos tradicional y más cercana al intercambio de experiencias ${ }^{37}$.

La gestión del conocimiento y capital humano es una de las más importantes interfaces de esta área, que permite crear oportunidades para la realización de trabajos con varios grados de complejidad. La formación debe ser discutida como elemento emancipador de los sujetos $^{38}$.

Todos los gestores participantes en la experiencia fueron enfáticos al resaltar la importancia del usuario de los servicios de salud y del trabajo en equipo como orientadores constantes de las prácticas profesionales del enfermero en la gestión.

El trabajo en equipo es una forma de trabajo colectivo en contraposición al modelo aislado e independiente, en el cual, muchos profesionales todavía están acostumbrados a trabajar. En el área de gestión en Enfermería, se hace decisivo para el éxito de las acciones que se desean implementar ${ }^{39}$.

Se discutió la importancia del modelo biomédico, que centralizaba el poder y la autonomía que fue minimizada con la implantación de un nuevo paradigma, descentralizador, que potencia las nuevas prácticas profesionales, con la búsqueda del trabajo en equipo y 
constante relación con la comunidad.

Definitivamente, el rumbo que debe ser seguido es la construcción de procesos de modificación de la atención centrada en el usuario, en sus necesidades, en sus fortalezas. Esto propicia un cambio profundo en el papel de los gestores y profesionales que pasan a planificar las actividades de una forma más descentralizada y orientada a las aspiraciones de la comunidad ${ }^{40-43}$.

\section{CONSIDERACIONES FINALES}

La experiencia hizo posible un debate significativo incluyendo cuestionamientos acerca del liderazgo, trabajo en equipo, comunicación, ética y reconocimiento profesional. El contacto con enfermeros provistos de éxito profesional en el área de gerencia fue esencial, complementados por los relatos de los alumnos graduandos que formularon múltiples posibilidades en el ejercicio de gestión en Enfermería.

Es interesante destacar que esta experiencia favoreció la integración de conocimientos entre la teoría y la práctica y contribuyó al reconocimiento de las dificultades y desafíos del porvenir. La teoría fue profundizada con las discusiones originarias de las prácticas de los profesionales participantes en las clases, fomentando articulaciones de prácticas extracurriculares y posibilidades de nuevas experiencias a los discentes del curso de graduación.

Los temas discutidos en clase enfatizaron las políticas nacionales de salud pública, así como el potencial del enfermero como gestor en los servicios de salud, sea en el ámbito público, privado o en el tercer sector.

Se destaca también la importancia de los discentes al haber experimentado una técnica de recopilación de datos que posteriormente será muy utilizada por ellos: la entrevista. Estos pudieron vivir las dificultades para hacerla y tuvieron idea acerca de la necesidad de instrumentalizarse para elaborar sus indagaciones y construir sus argumentaciones.

Se cree que el enfermero posee características inherentes a su formación que dibujan un perfil apropiado a la participación de este como miembro de la gestión del sistema de salud en sus más variados niveles de complejidad. Esto demuestra que la profesión posee características necesarias para el buen desarrollo del trabajo de un gestor, para que este sea capaz de aliviar las fragilidades y agregar esfuerzos para enaltecer las potencialidades del Sistema Único de Salud.

\section{REFERENCIAS}

1. Demo P. Pesquisa: princípio científico e educativo. Cortez; 2003.

2. Freire $P$. Pedagogia da autonomia: saberes necessários à prática educativa. $34^{a}$ ed. Rio de Janeiro: Paz e Terra; 2006.

3. Santos BS. A crítica da razão indolente: contra o desperdício da experiência. São Paulo: Cortez Editora; 2002.

4. Carvalho YM, Ceccim RB. Formação e educação em saúde: aprendizados com a Saúde Coletiva. In: Campos GWS, Minayo MCS, Akerman M, Drumond Júnior M, Carvalho YM, organizadores. Tratado de Saúde Coletiva. São Paulo - Rio de Janeiro: HUCITEC FIOCRUZ; 2006. p. 149-182. 
5. Mitre SM, Siqueira-Batista R, Mendonça JMG, Morais-Pinto NM, Meirelles CAB, Pinto-Porto $\mathrm{C}$, et al. Metodologias ativas de ensino-aprendizagem na formação profissional em saúde: debates atuais. Ciência Saúde Coletiva. 2008; 13(2): 2133-44.

6. Lourenção DCA, Benito GAV. Competências gerenciais na formação do enfermeiro. Rev Bras Enferm. 2010; 63(1): 91-7.

7. Ruthes RM, Cunha ICKO. Entendendo as competências para aplicação na enfermagem. Rev Bras Enferm. 2008; 61(1): 109-12.

8. Cunha ICKO, Ximenes Neto FRG. Competências gerenciais de enfermeiras: um novo velho desafio? Rev Texto Contexto Enferm. 2006; 15(3): 479-82.

9. Bagnato MHS, Rodrigues RM. Diretrizes Curriculares da Graduação de Enfermagem: pensando contextos, mudanças e perspectivas. Rev Bras Enferm. 2007; 60(5): 507-12.

10. Ito EE, Peres AM, Takahashi RT, Leite MMJ. O ensino de enfermagem e as diretrizes curriculares nacionais: utopia x realidade. Revista da Escola de Enfermagem da USP. 2006; 40(4): 570-5.

11. Brasil. Ministério da Saúde / Ministério da Educação e Cultura. Portaria Interministerial MS/MEC no 2.101, de 3 de novembro de 2005. Institui o Programa de Reorientação da Formação Profissional em Saúde - Pró-Saúde - para os cursos de graduação em Medicina, Enfermagem e Odontologia. Diário Oficial da União. Brasília. 2005.

12. Conselho Nacional de Educação. Câmara de Educação Superior. Resolução CNE/CES N. 3, de 07 de novembro de 2001. Institui as Diretrizes Curriculares Nacionais do curso de graduação em enfermagem. Diário Oficial da República Federativa da União. Brasília. 2001.

13. Brasil. Presidência da República. Lei no 9.394 de 20 de dezembro de 1996. Estabelece as diretrizes e bases da educação nacional. Diário Oficial da União. Brasília. 1996.

14. Waldow VR. Momento de cuidar: momento de reflexão na ação. Rev Bras Enferm. 2009; 62(1): 140-5.

15. Kurcgant P. Gerenciamento em Enfermagem. 2. ed. Rio de Janeiro: Guanabara Koogan; 2010.

16. Marquis BL, Huston CJ. Administração e liderança em enfermagem: teoria e prática. 6a ed. Porto Alegre: ArtMed; 2010.

17. Alves VLS. Gestão da Qualidade: Ferramentas Utilizadas no Contexto Contemporâneo da Saúde. São Paulo: Editora Martinari; 2009.

18. Dei Svaldi JC, Lunardi Filho WD, Gomes GC. Apropriação e uso de conhecimentos de gestão para a mudança de cultura na Enfermagem como disciplina. Rev Texto Contexto Enferm. 2006; 15(3): 500-7.

19. Pires MRGM, Spagnol CA, Brito MJM, Gazzinelli MFC, Montenegro LC. Diálogos entre a arte e a educação: uma experiência no ensino da disciplina de administração em saúde. Rev Texto Contexto Enferm. 2009; 18(3): 559-67.

20. Minayo MCS. O desafio do conhecimento: pesquisa qualitativa em saúde. $12^{a}$ ed. São Paulo: HUCITEC; 2010.

21. Rosa MVFPC, Arnoldi MAGC. A entrevista na pesquisa qualitativa: mecanismos para validação dos resultados. Belo Horizonte: Autêntica; 2006.

22. Britten N. Entrevistas qualitativas. In: Pope C, Mays N, organizadores. Pesquisa qualitativa na atenção à saúde. 3. ed. Porto Alegre: ArtMed, 2009. p. 23-31.

23. Gentil RC. O enfermeiro não faz marketing pessoal: a história explica por quê? Revista Brasileira de Enfermagem. 2009; 62(6): 916-18.

24. Melo CMM, Santos TA. A participação política de enfermeiras na gestão do Sistema Único de Saúde em nível municipal. Rev Texto Contexto Enferm. 2007; 16(3): 426-32.

25. Brasil. Ministério da Saúde. Secretaria Executiva. Departamento de Apoio à Descentralização. Coordenação-Geral de Apoio à Gestão Descentralizada. Diretrizes operacionais dos Pactos pela Vida, em Defesa do SUS e de Gestão. Brasília: Ministério da Saúde; 2006.

26. Cianciarullo TI. Instrumentos básicos para o cuidar: um desafio para a qualidade de 
assistência. Rio de Janeiro: Atheneu; 2000.

27. Carvalho GI, Santos L. Sistema Único de Saúde: comentários à Lei Orgânica da Saúde. 4ª̣ . ed. Campinas: Editora da UNICAMP; 2006.

28. Brasil. Ministério da Saúde. Secretaria de Atenção à Saúde. Departamento de Atenção Básica. Política Nacional de Atenção Básica. Brasília: Ministério da Saúde; 2006.

29. Brasil. Ministério da Saúde. Política Nacional de Promoção da Saúde. Brasília: Ministério da Saúde; 2006.

30. Fernandes MC, Barros AS, Silva LMS, Nóbrega MFB, Silva MRF, Torres RAM. Análise da atuação do enfermeiro na gerência de unidades básicas de saúde. Rev Bras Enferm. 2010; 63(1): 11-5.

31. Weirich CF, Munari DB, Mishima SM, Bezerra ALQ. O trabalho gerencial do enfermeiro na rede básica de saúde. Rev Texto Contexto Enferm. 2009; 18(2): 249-57.

32. Franco TB, Andrade CS, Ferreira VSC, organizadores. A produção subjetiva do cuidado: cartografias da Estratégia Saúde da Família. São Paulo: HUCITEC; 2009.

33. Trevizan MA, Mendes IAC, Hayashida M, Godoy S, Nogueira MS. The search for attitudinal commitment: tendency in the manager nurse's ethical behavior. Revista da Escola de Enfermagem da USP. 2009; 43(3): 712-16.

34. Feldman LB, Ruthes RM, Cunha ICKO. Criatividade e inovação: competências na gestão de enfermagem. Rev Bras Enferm. 2008; 61(2): 239-42.

35. Ruthes RM, Cunha ICKO. Gerenciamento de Enfermagem e administração das organizações do Terceiro Setor. Rev Bras Enferm. 2006; 59(6): 796-9.

36. Balsanelli AP, Jericó $M C$. Os reflexos da gestão pela qualidade total em instituições hospitalares brasileiras. Acta Paul Enferm. 2005; 18(4): 397-402.

37. Morreti-Pires RO, Bueno SMV. Relação docente-discente em Enfermagem e problemas na formação para o Sistema Único de Saúde. Acta Paul Enferm. 2009; 22(5): 645-651.

38. Ruthes RM, Cunha ICKO. Competências do enfermeiro na gestão do conhecimento e capital intelectual. Rev Bras Enferm. 2009; 62(6): 901-5.

39. Peduzzi M, Ciampone MHT. Trabalho em equipe e processo grupal. In: Kurcgant P. Gerenciamento em Enfermagem. Rio de Janeiro: Guanabara Koogan; 2005. p. 108-24.

40. Cunha GT. A construção da clínica ampliada na Atenção Básica. 3a ed. São Paulo: HUCITEC; 2010.

41. Ruthes RM, Feldman LB, Cunha ICKO. Foco no cliente: ferramenta essencial na gestão por competência em enfermagem. Rev Bras Enferm. 2010; 63(2): 317-21.

42. Campos GWS. Um método para análise e co-gestão de coletivos. 3. ed. São Paulo: HUCITEC; 2007.

43. Merhy EE, Magalhães Júnior HM, Rimoli J, Franco TB, Bueno, WS, organizadores. O

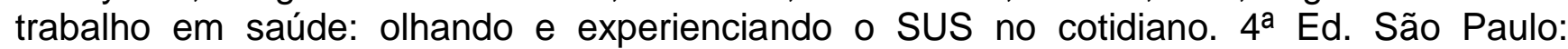
HUCITEC, 2007.

ISSN 1695-6141

๑ COPYRIGHT Servicio de Publicaciones - Universidad de Murcia 